\title{
MOST Moderate-Weak Inversion Region as the Optimum Design Zone for CMOS 2.4-GHz CS-LNAs
}

\author{
Rafaella Fiorelli, Fernando Silveira and Eduardo Peralías
}

\begin{abstract}
In this paper, the MOS transistor (MOST) moderate-weak inversion region is shown to be the optimum design zone for CMOS 2.4-GHz common-source low noise amplifiers (CS-LNA) focused on low power consumption applications. This statement is supported by a systematic study where the MOST is analyzed in all-inversion regions using an exhaustive CS-LNA noise figure-power consumption optimization technique with power gain constraint. Effects of bias choke resistance and MOST capacitances are carefully included in the study to obtain more accurate results, especially for the moderate-weak inversion region. Noise figure, power consumption and gain versus the inversion region are described with design space maps, providing the designer with a deep insight of their trade-offs. The Pareto-optimal design frontier obtained by calculation, -showing the moderate-weak inversion region as the optimum design zone- is re-verified by extensive electrical simulations of a high number of designs. Finally, one 90-nm 2.4-GHz CS-LNA Pareto optimal design is implemented. It achieves the best FoM considering under-mW CS-LNAs published designs, consuming $684 \mu \mathrm{W}$, a noise figure of $4.36 \mathrm{~dB}$, a power gain of $9.7 \mathrm{~dB}$ and an IIP3 of $-4 \mathrm{dBm}$ with load and source resistances of $50 \Omega$.
\end{abstract}

\section{Index Terms}

CS-LNA, Moderate Inversion, Weak Inversion Noise figure, Optimization, Low power, $g_{m} / I_{D}$, Design Methodology, Pareto optimal.

\section{INTRODUCTION}

The increase of radiofrequency (RF) applications focused on low power, short range and low rate, such as those that meet ZigBee or low-energy Bluetooth standards, are forcing the industry to develop low-cost chip solutions. This cost reduction is mainly achieved by using CMOS technology and reaching shorttime-to-market designs, where a prior knowledge of the circuits behavior considerably helps. Biasing the 
MOST in weak (WI) and moderate (MI) inversion region instead of strong inversion (SI) is necessary to achieve the mentioned power reduction of orders of magnitude [1]; however, the effect of moving to MI and WI means an increment in the parasitic capacitances and a reduction in the transition frequency $f_{T}$, hence, in the maximum working frequency. For the nanometer CMOS technologies used today, it is possible to bias the MOST in MI-WI region when working in the GHz-range, where the conservative quasi-static limit of one-tenth of $f_{T}$ is yet valid. Various CMOS analog RF designs biased in MI and WI have been reported, as shown in [2]-[11], among others, where a considerable power reduction is achieved compared with biasing in strong inversion. However, there is a lack of published works that systematically study all-inversion regions of MOST for RF design, going farther than just showing the feasibility of a particular design and find the optimum design zone for certain constraints. This deficiency is notorious in LNAs, as in CS-LNAs.

In this paper, we consider the CS-LNA optimum in the sense of Pareto noise figure-power consumption optimal frontier with gain constraint, input/output LNA impedance matching and without tight linearity requirements, to cope with the before mentioned standards. All regions of operation of the MOST are systematically analyzed to prove the narrowband RF under-mW $2.4 \mathrm{GHz}$ CS-LNAs optima lay in the moderate-weak inversion region. Finding this Pareto frontier without using electrical optimization tools implies the implementation of an optimization method that considers the inversion region as its core variable. In this sense, this paper follows the same approach of [10]-[20], where optimization techniques for RF circuits applied before electrical simulation are proven as a suitable design strategy. Particularly for CS-LNA, well-known optimization techniques have been presented, as in [12]-[14], but these techniques only cover the strong inversion region, do not study the effects of CS-LNA output impedance in the optimization process and disregard the inductors parasitics. Our approach, which expands previous studies, helps the designer to give a deep insight into the behavior of low-power CS-LNAs fundamental characteristics as well as their trade-offs, which helps in reducing consumption without losing gain, noise or linearity performance.

To demonstrate that optimal designs of 2.4-GHz CS-LNAs are in the moderate-weak inversion region, we need to systematically study the MOST in all regions of operation: weak, moderate and strong inversion, finding the best design in each case. Although the authors know that more efficient optimization algorithms exist to cope with multi-objective optimization, our approach allows us not only to find the Pareto frontier but also to study in detail the evolution of the LNA characteristics in all regions as well as the arising compromises, as we will see later on.

The use of the $g_{m} / I_{D}$ ratio [21], [22] is our fundamental tool to cover all-inversion regions of the 
MOST. Its election is principally due to it being a good indicator of the inversion region because of the very small range of $g_{m} / I_{D}$ values, as they are in the order of $1 \mathrm{~V}^{-1}$ to $30 \mathrm{~V}^{-1}$ for a nanometer bulk MOST. In fact, as a rule of thumb [23], for our RF 90-nm CMOS 1-Poly 9-Metals process, called from now on Reference Technology, WI is considered for $g_{m} / I_{D}$ values well above $20 \mathrm{~V}^{-1}$, SI is well below $g_{m} / I_{D}=10 V^{-1}$ and MI is in the midst of them. Also, the $g_{m} / I_{D}$ ratio of a saturated MOST has a biunivocal relation with the normalized current or current density, defined as $i=I_{D} /(W / L)$ [21], with $I_{D}, W$ and $L$ the MOST drain current, width and length, respectively. Since $i$ versus $g_{m} / I_{D}$ is quasi-independent of $V_{D S}$ and $W$ for $L$-constant MOST (see Fig. 1(a)), given $g_{m} / I_{D}$ and $I_{D}$, the size of MOST is fixed.

The proposed approach considers input/output LNA impedance matching to find the MOST operation point and all components' geometries that minimize its noise figure for each quiescent current $I_{D}$, with a specific power gain constraint. In this paper, the design method used follows the guidelines the authors enunciate in [10]; i.e. the application of semi-empirical modeling for RF devices (in particular MOST and inductors) and the use of analytical expressions of circuit's small-signal model in a developed ad-hoc design flow which arrange them together with the necessary decisions fixed by LNA specifications and technological constraints.

The search of the optimum in all-inversion regions needs a reasonably accurate MOST model, especially in MI and WI regions. It oblige us to use not only the intrinsic capacitances (considered for this study only the five quasi-static capacitances ${ }^{1}$ ), but also the extrinsic ones, even the generally disregarded, as $C_{d s}$. Analogously, for passive components such as inductors, their parasitics cannot be ignored in a first approximation. Additionally, noise figure computation takes into account noise parameters variations with the MOST transistor inversion region and includes the noise due to bias choke resistor. Moreover, to correctly compute power gain, an accurate value of the CS-LNA output impedance is deduced. Finally, the layout effects are incorporated in the carried out optimization because semi-empirical models of the used RF devices contain this information from the foundry.

To sum up, the new ideas of this paper are the following: (1) to show that the optimum design zone for CS-LNAs, for $2.4 \mathrm{GHz}$ ISM band, is the MOST moderate-weak inversion region; (2) the systematical study of the design trade-offs among power consumption, noise, gain and element sizing among all MOST inversion regions; (3) the assertion that good designs are obtained when working with low currents and

\footnotetext{
${ }^{1}$ The hypothesis of the MOST operation below the quasi-static limit of one tenth of the transition frequency $f_{T}$ [24] is valid for the considered working frequency and process.
} 

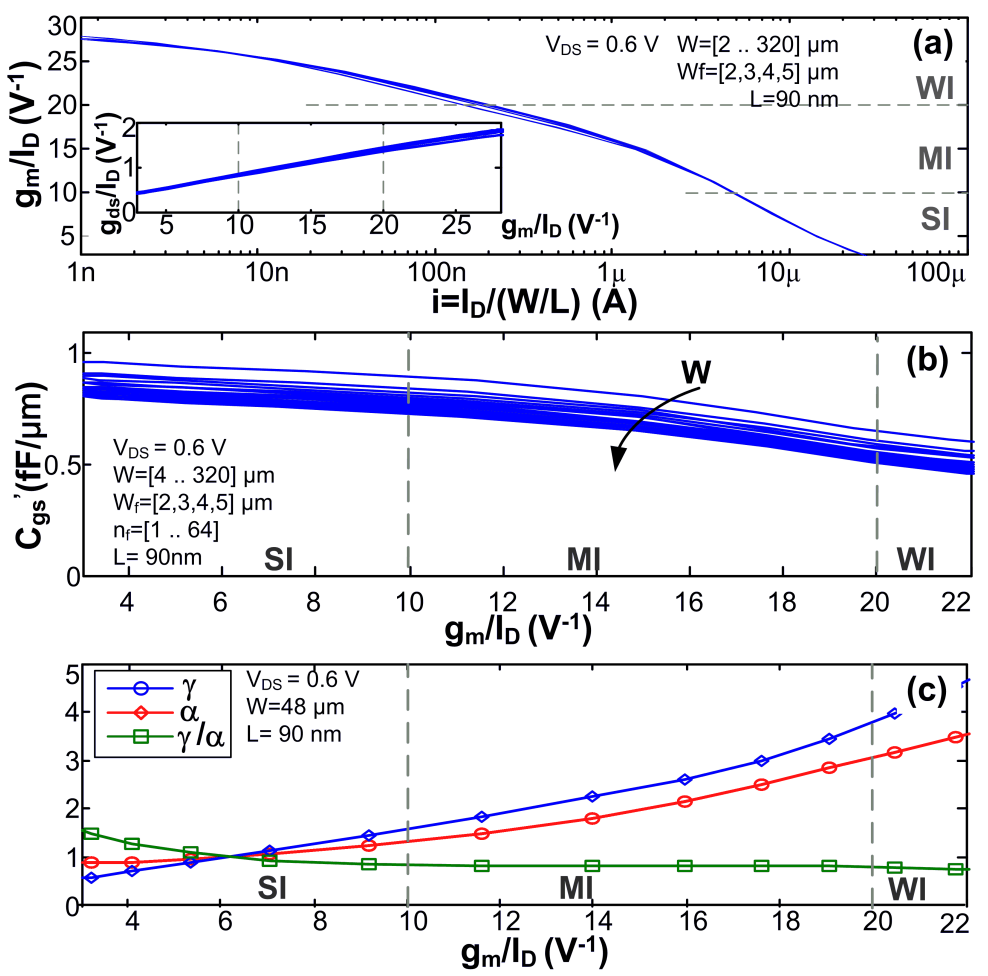

Fig. 1. (a) $i$ and $g_{d s} / I_{D}$ (inner plot) and (b) $C_{g s}^{\prime}$ for a wide set of nMOST finger widths $W_{f}$ and number of fingers $n_{f}$ versus $g_{m} / I_{D}$. (c) Typical noise parameters $\gamma, \alpha$ (and $\gamma / \alpha$ ) for all-inversion regions.

in MI-WI regions; (4) the need of have a limited set of parametrized models, simple yet accurate, for the circuit's elements (particularly for MOST and inductors).

The outline of this paper is as follows. Section II describes the MOST and passive components modeling. Section III provides the small-signal LNA analysis. Section IV details the CS-LNA optimization methodology and provides the Pareto optimal LNA design's frontier, also presents the validation technique via several CS-LNA designs and the gathered conclusions. Section V shows the measurements of a fabricated 2.4-GHz 90-nm CS-LNA. Finally, Section VI summarizes the main contributions of this paper.

\section{RF DEVICE MODELS}

\section{A. MOST model}

Modeling of MOST is done by using a semi-empirical model based on a lookup-table (LUT) [10], [22], [25] obtained by electrical DC and noise simulations, performed only once, on models qualified by the foundry of our Reference Technology. This way, it jointly considers second and higher order effects that appear in nanometer technologies, as discussed in [10]. The MOST model specifically includes the 
following characteristics as a function of $g_{m} / I_{D}$ : (1) normalized current $i$; (2) output conductance to current ratio $g_{d s} / I_{D}$; (3) normalized MOST capacitances $C_{i j}^{\prime}=C_{i j} / W$ with $i j=\{g s, g d, g b, b s, b d\}$; and (4) drain white noise parameters. The extrinsic capacitances and the gate resistance, $R_{g M O S}$, are considered constants for all inversion regions but dependent on the MOST geometry (e.g. in our case $R_{g M O S} \approx 4.3+78 / n_{f}$, with $n_{f}$ the MOST number of fingers). The capacitance normalization of item (3) can be performed because intrinsic capacitances as well as the extrinsic part of gate-source and gate-drain ones are proportional to $W$, and MOST length is always fixed equal to the minimum $L_{\min }=90 \mathrm{~nm}$ (the extrinsic part of the gate-bulk capacitance is disregarded). Features (1) and (2) are presented in Fig. 1(a). An example of item (3) is in Fig. 1(b), where $C_{g s}^{\prime}$ is plotted for a wide range of MOST widths. Its spread for a fixed inversion level is around $10 \%$, which is acceptable for our purposes. For the rest of normalized capacitances, the variation is below this value $\left(C_{g d}^{\prime} \approx(0.36 \pm 0.03) \mathrm{fF} / \mu \mathrm{m}, C_{g b}^{\prime}<(0.03 \pm 0.03) \mathrm{fF} / \mu \mathrm{m}\right.$, $C_{d s}^{\prime} \approx 0.09 \mathrm{fF} / \mu \mathrm{m}$, and $\left.C_{b s}^{\prime} \approx C_{b d}^{\prime} \approx 0.34 \mathrm{fF} / \mu \mathrm{m}\right)$. All LUTs capacitances are estimated by electrical DC simulations. The second-order dependence on $V_{D S}$ voltage of items (2) and (4) is also considered in the correspondent LUTs. Parameter $g_{m b}$ is also modeled, being its dependence practically linear with $g_{m} / I_{D}\left(g_{m b} / I_{D} \approx 0.13 g_{m} / I_{D}\right)$.

MOST drain white noise is the CS-LNA highest noise contributor at the working frequencies. Its power spectral density (psd) is modeled with $\overline{i_{w, d}^{2}}=4 k_{B} T \frac{\gamma}{\alpha} g_{m}$, with $k_{B}$ the Boltzmann constant, $T$ the absolute temperature, $\gamma$ the excess noise factor [12] and $\alpha=g_{m} / g_{d 0}$, being $g_{d 0}$ the zero- $V_{D S}$ bias drain conductance [24]. Extraction of $\gamma, \alpha$ and the relation $\gamma / \alpha$ versus $g_{m} / I_{D}$ via electrical simulations generates the curves of Fig. 1.(c). In this case the generally used values of $\gamma \cong 2 / 3$ and $\alpha \cong 0.6$ do not adjust to the extracted data, in particular for MI and WI regions. Additionally, the induced-gate noise psd is modeled as $\overline{i_{g}^{2}}=\frac{16}{5} \pi^{2} k_{B} T \alpha \delta \frac{C_{g s}^{2}}{g_{m}} f^{2}$, where $\delta$ is the gate noise coefficient [12], considered here an independent parameter equal to $4 / 3$.

\section{B. Passive Components models}

Three types of passive components are used in our designs: inductors, capacitors and resistors. For our Reference Technology, we obtain their models just once from AC electrical simulations at the working frequency $\omega_{0}=2 \pi f_{0}$, saving them in LUTs. The simulation extracts the equivalent element's value together with their equivalent parasitics, from which their quality factors are computed. To speed-up the design flow, all passive elements are selected from a parameterized library provided and characterized by the foundry up to $20 \mathrm{GHz}[25]$.

Inductor model consists of an equivalent inductor with a parasitic resistor, either in parallel or in series, 


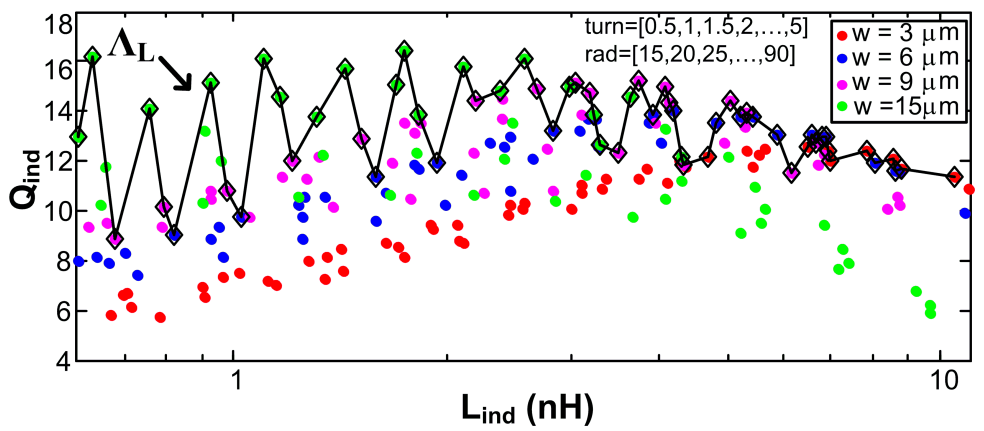

Fig. 2. Inductor quality factors $Q_{\text {ind }}$ for $f_{0}=2.45 \mathrm{GHz}\left(\mathrm{w}=\right.$ width). $Q_{\text {ind }}^{\max }$ are marked with a black line, and selected for the inductor LUT $\Lambda_{L}$.

whose data are related through a LUT. To find this LUT, firstly a large set of inductors is simulated, varying their physical magnitudes (turns, coil width and radius), and obtaining their characteristics, i.e. inductance $L_{\text {ind }}$, parasitic parallel and serial resistances, $R_{i n d}^{(p)}$ and $R_{i n d}^{(s)}$ and quality factor $Q_{\text {ind }}=\omega_{0} L_{\text {ind }} / R_{\text {ind }}^{(s)} \cong$ $R_{i n d}^{(p)} / \omega_{0} L_{i n d}$, as it is shown in the scatter plot of Fig. 2. Secondly, the inductor LUT $\Lambda_{L}$ is collected, where for each inductance value $L_{i n d}$ we find the inductor with the highest quality factor $Q_{i n d}^{\max }$ (black line of Fig. 2) and the geometry of its implementation. The characterization is made dense enough to cover the whole range of feasible inductances $\left(L_{i n d} \in[0.2,11.5] \mathrm{nH}\right)$ with a logarithmic grid. This fact will be justified in Section III. For the Reference Technology and from Fig. 2, we can extract that, at $f_{0}=2.4 \mathrm{GHz}, R_{\text {ind }}^{(p)} \approx 200 \times 10^{9} L_{\text {ind }}$ and $R_{\text {ind }}^{(s)} \approx 1.2 \times 10^{9} L_{\text {ind }}$, considering that $Q_{\text {ind }}^{\max }$ is approximately constant to 13 in whole range.

MiM Capacitors and Poly resistors are modeled in an equivalent way to the inductors, in ranges [10 fF, $2 \mathrm{pF}]$ and $[2,100] \Omega$, respectively. Resistor semi-empirical model assumes as parasitic a series reactance $X_{\text {res. }}$. Since in the adopted ranges and at $f_{0}=2.4 \mathrm{GHz}$, capacitor quality factor are above one hundred, and resistor quality factors $\left(Q_{r e s}=R_{\text {res }} /\left|X_{\text {res }}\right|\right)$ are above one thousand, we can consider them, in first approximation, as ideal components.

\section{LNA SYNTHESIS}

Schematic of Fig. 3 depicts the CS-LNA basic topology adopted in this work to prove our hypothesis on its optimal behavior when MOS transistors are in moderate-weak inversion region. Its simplicity together with its widespread use in multiple low-power implementations and applications justify its choice as our reference topology. This section shows the small-signal analytical description carried out on this topology for later use in the optimization process. 


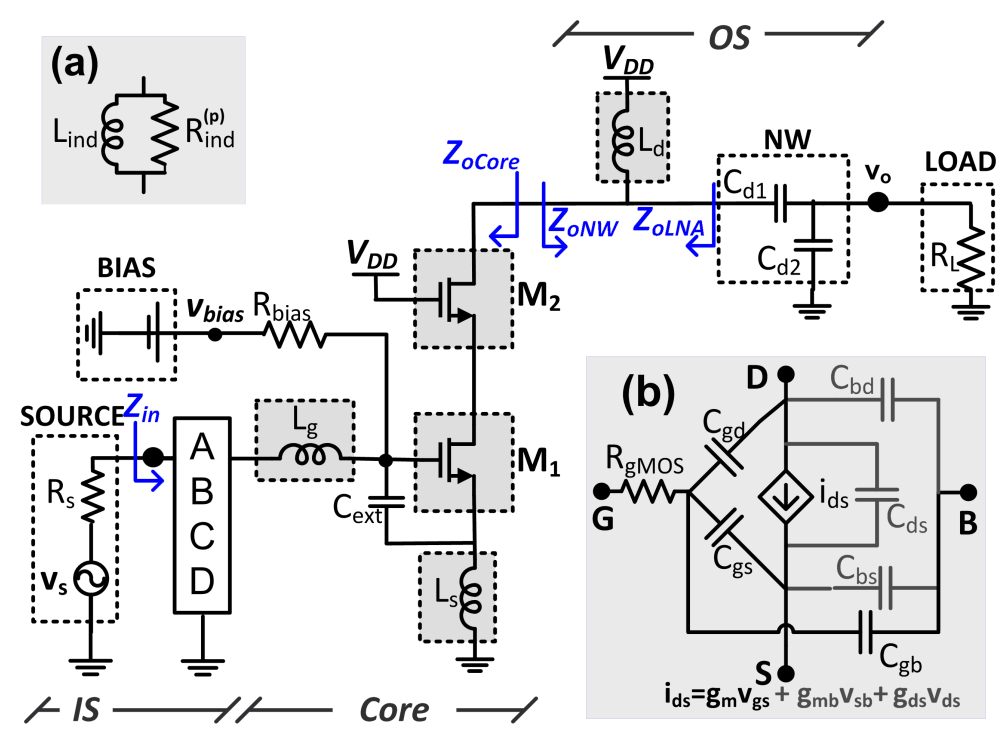

Fig. 3. Topology of the CS-LNA, including (a) inductor model and (b) MOST models. IS, Core and OS denote the Input, Core and Output stages.

The Core of the LNA consists of the gate and source inductors, $L_{g}$ and $L_{s}$, the capacitor $C_{\text {ext }}$, and the MOS transistors: $M_{1}$ as amplifier, and $M_{2}$ as cascode; $M_{2}$ decouples input and output signals and increases the output impedance. For simplification, $M_{2}$ is considered equal to $M_{1}$ (same geometry), and since they have the same current, they reach the same current density $i$, and hence the same $g_{m} / I_{D}$. In this topology, we take advantage of an external gate-source capacitor $C_{e x t}$ to decouple the LNA's power gain from noise [15] and reducing all $R_{g M O S}$ effects. Device $R_{b i a s}$ is a choke resistor for the gate bias voltage and a priori it is not neglected, and it will be taken into account in the amplifier analysis. Bias voltage is obtained via a current mirror where output branch is a replica of the LNA $M_{1}-M_{2}$ configuration.

As input stage (IS), we assume a known passive network, basically including the capacitances of the input pad and ESD protection diodes, the blocking capacitor, and the inductance of bonding wire. This block is modeled with a transmission ABCD two-port network where to its input is a signal from an ideal source $v_{s}$ with resistive impedance $R_{s}(=50 \Omega)$ at the working frequency $f_{0}$. The output stage (OS) is a matching network between the core stage and the load $R_{L}$, that comprises the pull-up drain inductor $L_{d}$ and a capacitive network NW, $C_{d 1}$ and $C_{d 2}$. Without loss of generality, load $R_{L}$ is assumed purely resistive. Impedance seen at the input of OS is denoted by $Z_{O N W}$.

Because the LNA input impedance is fixed, the design procedure applies the well-known input matching technique [13], which is suitable for low-power applications. Thus, $Z_{i n}\left(f_{0}\right)=R_{s}$, where $Z_{i n}$ is the LNA 
input impedance, which includes the effects of the $\mathrm{ABCD}$, Core and OS, i.e. $Z_{i n}\left(M_{1}, M_{2}, L_{g}, C_{e x t}, L_{s}, L_{d}, C_{d 1}, C_{d 2} ; f_{0} ; R_{b}\right.$

Inset (a) of Fig. 3 describes the inductors' model utilized in this analytical study: its inductance $L_{\text {ind }}$ jointly with the equivalent parallel resistance $R_{i n d}^{(p)}$ (taken from LUT $\Lambda_{L}$ detailed in Section II). Inset (b) of Fig. 3 represents the considered model for MOS transistors. All MOST characteristics are known when values of $g_{m} / I_{D}$ and $I_{D}$ are given, by means of LUTs as explained in Section II. Gate resistance $R_{g M O S}$ is not neglected in the model as its effects are noticeable for small-width MOST (its reduction is reached by increasing the number of fingers $n_{f}$ of MOST).

Even though a simpler model for the MOST could simplify the design procedure, especially in SI and SI-MI regions $\left(g_{m} / I_{D}<15\right)$, we must describe correctly the LNA behavior up to WI while reaching a good precision in the MI-WI zone $\left(g_{m} / I_{D}<20\right)$. In these regions, MOSTs are so wide that parasitics, $C_{g s}, C_{g d}, C_{g b}, C_{b s}, C_{b d}$ and $C_{d s}$, are not negligible, and consequently direct coupling between input and output stages must be accurately modeled.

To achieve the input-matching goal, we need precise evaluation of LNA input impedance, $Z_{i n}$, Core output impedance, $Z_{o C o r e}$, and input impedance of OS, $Z_{O N W}$. Either rational algebraic expressions obtained via a symbolic analysis tool or subroutines that solve the complete linearized circuit are two alternative procedures that we have successfully tested.

For the design process, we apply the $g_{m} / I_{D}$ method of [21] so the pair $\left(g_{m} / I_{D}, I_{D}\right)$ are the two first input design variables. This enable the visualization of the optimum zone and the consumption trade-off with other CS-LNA characteristics. The last input design variable is $L_{g}$, taken from the LUT $\Lambda_{L}$ in order to use only the best inductors available in the Reference Technology and to know in advance their parasitics.

The unknowns to be found are $W, L_{s}, C_{e x t}, L_{d}$ and $C_{d 1}, C_{d 2}$. To obtain them, we numerically solve the equation system $Z_{i n}=R_{s}$, following the next Iterative Procedure, efficiently implemented using a numerical solver in Matlab environment:

1) Fix values of $\left(g_{m} / I_{D}, I_{D}\right)$ and $L_{g}$. From the pair $\left(g_{m} / I_{D}, I_{D}\right)$ and the definition of $i, W$ is deduced from the LUTs of $g_{m} / I_{D} @ \mathrm{i}$.

2) Determine two seed values for $L_{s}$ and $C_{\text {ext }}$, from the closed expression in (7) corresponding to the LNA simplified scheme of Appendix A. Since both must be feasible in the technology, $L_{s}$ should be discretized to the nearest value in $\Lambda_{L}$.

3) In this moment whole input and Core stages are fully known, i.e. $L_{g}, C_{e x t}, L_{s}$, and $W$. Then, calculate the core output impedance $Z_{o \text { Core }}\left(f_{0}\right)$.

4) Determine an output matching network, $L_{d}$ and $C_{d 1}, C_{d 2}$, such as $Z_{o C o r e}\left(f_{0}\right)=Z_{o N W}^{*}\left(f_{0}\right)$, where 
the asterisk $\left(^{*}\right)$ indicates the complex conjugate operator. This network must be feasible within the technology and some directions to obtain it are pointed in next subsection.

5) As now the whole LNA elements are known, compute $Z_{i n}\left(f_{0}\right)$.

6) Compare $Z_{i n}$ with the source impedance $R_{s}$, computing $S_{11}\left(=\left|Z_{i n}-R_{s}\right| /\left|Z_{i n}+R_{s}\right|\right)$, and decide if the solution is good enough, e.g. if $S_{11}<-20 \mathrm{~dB}$. If not, correct initial values of $L_{s}$ (within $\Lambda_{L}$ ) and $C_{\text {ext }}$ by a minimization in least-squares sense, and return to Step 3).

\section{A. Power gain}

An efficient CS-LNA power transfer needs an effective load of the Core stage with a high resistive term. Since in general the output impedance $Z_{o \text { Core }}$ is capacitive, $\left(\operatorname{Im}\left(Z_{o \text { Core }}\right)<0\right), L_{d}$ is used to produce a purely inductive output impedance, $Z_{o L N A}\left(=Z_{o C o r e} \| Z_{L d}\right)$, that via the capacitive network NW is matched to $R_{L}$. This impedance transformation becomes easier when the drain inductor value is lower, hence when the design area is smaller. However, in that case the parallel parasitic resistance, $R_{d}^{(p)}$, also decreases, implying a reduction in the output power or equivalently in the LNA power gain. Thus, $L_{d}$ value is a trade-off between these two conditions: acceptable area and high power gain. Mathematically, requirements needed to find a network NW are $\operatorname{Re}\left(Z_{o L N A}\right) \leq R_{L}$ and $\operatorname{Im}\left(Z_{o L N A}\right)>0$, which produce the following value for $L_{d}$ :

$$
L_{d}=\frac{1}{\omega_{0}\left(\operatorname{Im}\left(1 / Z_{o L N A}\right)+\sqrt{G_{o L N A}\left(1 / R_{e f f L}-G_{o L N A}\right)}\right)},
$$

where $R_{e f f L}=\operatorname{Re}\left(Z_{o L N A}\right)$ and

$$
G_{o L N A}=\operatorname{Re}\left(\frac{1}{Z_{\text {oLNA }}}\right)=\operatorname{Re}\left(\frac{1}{Z_{\text {oCore }}}\right)+\frac{1}{R_{d}^{(p)}}
$$

Since $L_{d}$ in (1) depends on $G_{o L N A}$ in (2), and this one depends on $L_{d}$ through its parasitic $R_{d}^{(p)}$, the value of $L_{d}$ must be obtained in an iterative way. Also, it must verify, $L_{d} \leq L_{i n d}^{\max }$, where $L_{\text {ind }}^{\max }$ is the maximum value for a feasible inductor in the technology. This way, we have to reduce the constraint value $R_{\text {effL }}$ until that feasibility condition is verified.

Assuming that input and output matching conditions are met, the power gain is:

$$
G=10 \log \left(\frac{v_{o}^{2} / R_{L}}{v_{s}^{2} / 4 R_{s}}\right)=10 \log \left(4 \frac{R_{s}}{R_{L}} A_{v}^{2}\right) .
$$

where $A_{v}$ is the total voltage gain of CS-LNA from primary input $v_{s}$ to the voltage $v_{o}$ in the load $R_{L}$. This value is available in the linearized circuit when it is fully determined. 
Due to the high sensitivity of $G$ with $L_{s}$, and being that approximately logarithmic, $\frac{\partial G}{\partial \log \left(L_{s}\right)} \approx-20 \mathrm{~dB}$, we have used a logarithmic grid in the generation of the inductor LUT $\Lambda_{L}$ to control the maximum shift of power gain when two adjacent inductors are possible choices for the source inductor. In Fig. 2 the used logarithmic grid of 80 point per decade implies a maximum $\Delta G=0.25 \mathrm{~dB}$.

\section{B. Noise figure}

For the evaluation of noise figure, $N F$, of the CS-LNA in Fig. 3, we have considered five main noise sources: choke resistor, $R_{\text {bias }}$, gate and drain inductors, $L_{g}, L_{d}$, and transistors, $M_{1}$ and $M_{2}$. Concrete terms and expressions for these sources are given in Appendix B.

\section{OPTIMUM DESIGN ZONE VALIDATIONS AND DESIGN TRADE-OFFS}

This section proves our hypothesis of the location of the Pareto-optimal design frontier in the MI-WI region. We systematically obtain the set of feasible CS-LNA designs that are optimal in the Pareto sense for the trade-off between minimum noise figure and minimum power consumption and constrained to a fixed power gain. For the sake of simplicity in description, we implement the optimization process as an exhaustive search method synthesizing each feasible LNA with the Iterative Procedure of Section III. It covers the full design domain, selected in this case as the set of all possible drain currents $\left(I_{D}\right)$ for all possible MOST inversion levels (defined with the $g_{m} / I_{D}$ ratio).

The LNA Pareto frontier is found by means of using an optimization flow, called Exhaustive Optimization Process (EOP). For each $I_{D}$, the EOP provides with the optimum $g_{m} / I_{D}$ (and its corresponding $N F$ and $G$ ) and all possible CS-LNA designs for all-inversion regions in the whole range of $g_{m} / I_{D}$. To assess the results of EOP, it was implemented in Matlab computational routines ${ }^{2}$; its details are discussed in depth in Appendix C.

To study the behavior of the LNA characteristics against the inversion region, we generate a family of curves and contour maps considering all the databases obtained from the Reference Technology, for $f_{0}=2.45 \mathrm{GHz}, L_{\text {min }}=90 \mathrm{~nm}, R_{\text {bias }}=1 \mathrm{k} \Omega, R_{s}=R_{L}=50 \Omega$, and with a very simple ABCD network consisting of a blocking capacitor of $100 \mathrm{pF}$. First, the $N F$ and $G$ are studied. Their family of curves are depicted in Fig. 4 and their contour maps are plotted in Fig. 5, considering $g_{m} / I_{D}$ varying in $[5,21] \mathrm{V}^{-1}$ and $I_{D}$ in $[0.4,1.4] \mathrm{mA}$, with a grid linearly spaced of $0.5 \mathrm{~V}^{-1}$ and $0.1 \mathrm{~mA}$, respectively. No restrictions are applied for $N F$ and $G$ in order to observe their behavior in the whole design domain. These plots

\footnotetext{
${ }^{2}$ The EOP can also be implemented in IC design environments, as Cadence or Synopsys, assisted by RF electrical simulators.
} 


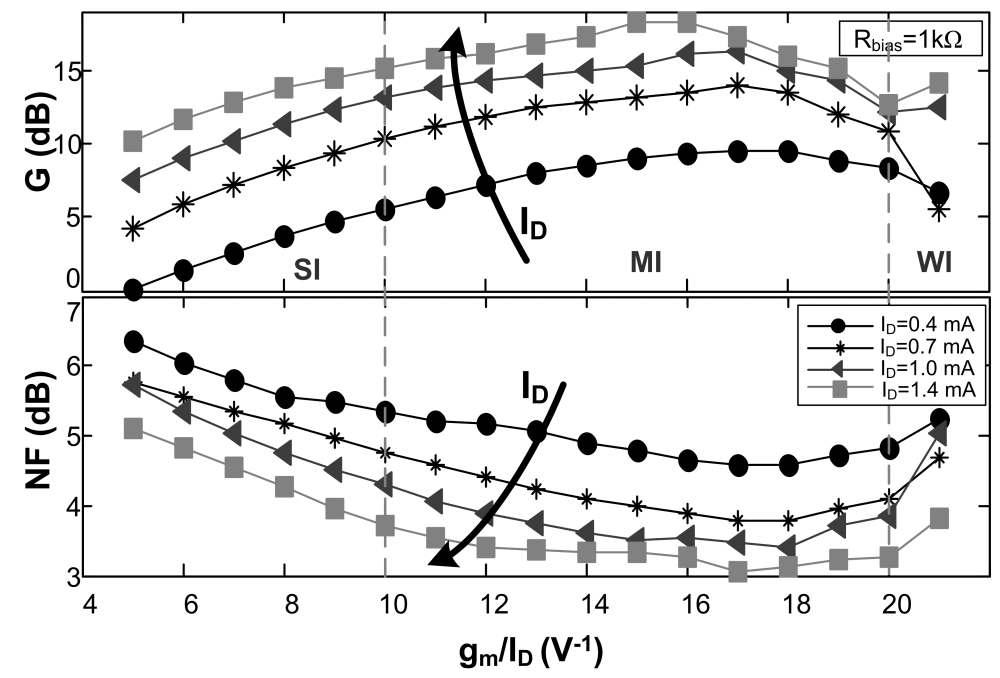

Fig. 4. (a) $G$ and (b) $N F$ for all-inversion regions and for four drain currents obtained applying the EOP without restrictions.

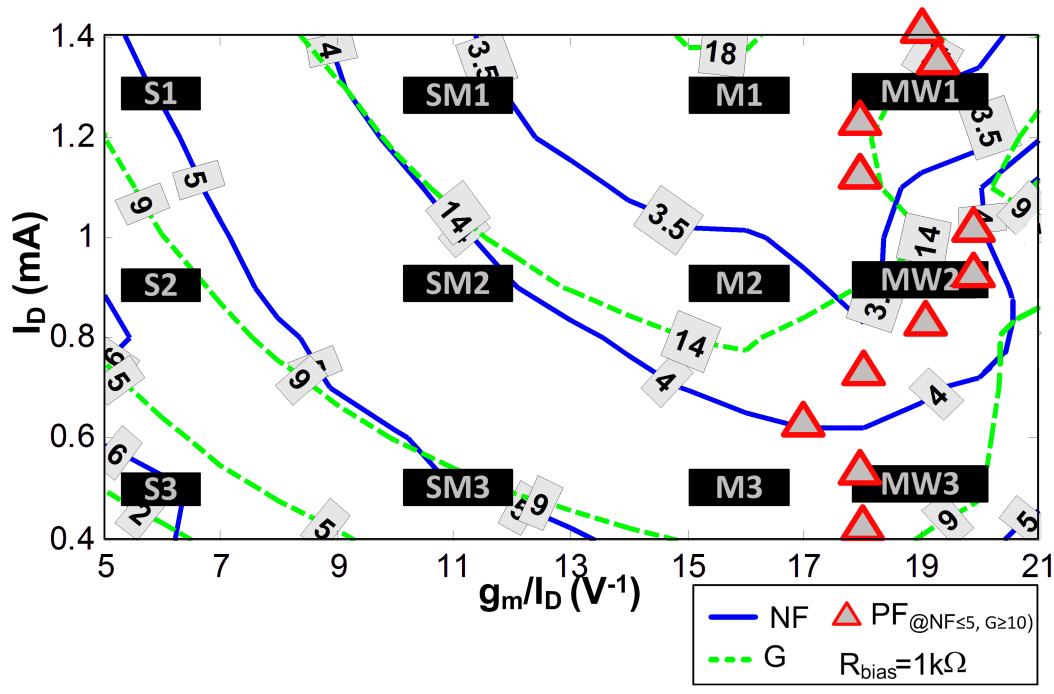

Fig. 5. Design space map of $N F$ and $G$ vs. $I_{D}$ and $g_{m} / I_{D}$ without restrictions. Design points used in the methodology validation as well as the Pareto optimal frontier $(\mathrm{PF})$ are highlighted.

show the expected trade-off of $N F$ and $G$ versus current consumption: raising $I_{D}$ implies a reduction in $N F$ and an increment in $G$, and vice versa. Besides, when the $g_{m} / I_{D}$ is swept, the best values of $N F$ and $G$ are always obtained in $M I$, reaching a reduction of almost $1 \mathrm{~dB}$ in the $N F$ and an improvement of almost $10 \mathrm{~dB}$ in the $G$ when compared with the figures of SI and WI; a feature not shown in prior works. 
TABLE I

METHOD VALIDATION: COMPARISON BETWEEN COMPUTATIONAL ROUTINES AND SCHEMATIC SIMULATIONS.

\begin{tabular}{|l|c|c|c|c|c|c|c|c|c||c|c|c|c|c|}
\hline Design & $g_{m} / I_{D}$ & $I_{D}$ & \multicolumn{2}{|c|}{$\mathrm{G}(\mathrm{dB})$} & \multicolumn{2}{|c|}{$\mathrm{NF}(\mathrm{dB})$} & $\mathrm{IIP} 3$ & $S_{11}$ & $S_{22}$ & $\mathrm{~W}$ & $C_{e x t}$ & $L_{s}$ & $L_{g}$ & $L_{d}$ \\
& $(1 / \mathrm{V})$ & $(\mathrm{mA})$ & Calc. & Sim. & Calc. & Sim. & $(\mathrm{dBm})$ & $(\mathrm{dB})$ & $(\mathrm{dB})$ & $(\mu \mathrm{m})$ & $(\mathrm{fF})$ & $(\mathrm{nH})$ & $(\mathrm{nH})$ & $(\mathrm{nH})$ \\
\hline S1 & 6 & 1.3 & 11.0 & 10.2 & 5.0 & 4.9 & -5.3 & -16.1 & -37.2 & 11.5 & 354 & 0.5 & 10.4 & 10.4 \\
S2 & 6 & 0.9 & 8.2 & 7.4 & 5.4 & 5.6 & -4.5 & -16.2 & -41.8 & 8.0 & 353 & 0.6 & 10.4 & 10.4 \\
S3 & 6 & 0.5 & 3.0 & 2.4 & 5.6 & 6.0 & -2.2 & -17.8 & -35.0 & 4.4 & 388 & 3.7 & 6.6 & 10.4 \\
SM1 & 11 & 1.3 & 15.0 & 14.5 & 3.5 & 3.4 & -7.5 & -17.7 & -35.2 & 33.1 & 376 & 0.63 & 8.8 & 10.4 \\
SM2 & 11 & 0.9 & 13.2 & 12.7 & 4.2 & 4.0 & -7.3 & -15.6 & -40.9 & 22.9 & 340 & 0.3 & 10.4 & 10.4 \\
SM3 & 11 & 0.5 & 8.4 & 7.9 & 5.1 & 4.9 & -6.0 & -16.2 & -39.4 & 12.7 & 348 & 0.6 & 10.4 & 10.4 \\
M1 & 16 & 1.3 & 17.8 & 16.5 & 3.3 & 3.1 & -8.5 & -17.1 & -13.9 & 124 & 251 & 0.4 & 8.8 & 10.4 \\
M2 & 16 & 0.9 & 14.9 & 13.9 & 3.5 & 3.3 & -2.3 & -17.8 & -17.8 & 85.8 & 317 & 0.63 & 8.6 & 10.4 \\
M3 & 16 & 0.5 & 11.3 & 10.7 & 4.3 & 4.2 & -8.9 & -16.6 & -21.0 & 50 & 304 & 0.4 & 10.4 & 10.4 \\
MW1 & 19 & 1.3 & 14.4 & 12 & 2.8 & 3.0 & -3.7 & -18.8 & -8.5 & 420 & 66 & 0.8 & 5.9 & 6.6 \\
MW2 & 19 & 0.9 & 14.0 & 12.0 & 3.5 & 3.6 & -16.4 & -18.8 & -6.7 & 291 & 107 & 0.4 & 7.8 & 7.8 \\
MW3 & 19 & 0.5 & 11.1 & 9.5 & 4.2 & 4.2 & -11.5 & -17.9 & -9.4 & 162 & 232 & 0.5 & 8.8 & 10.4 \\
\hline
\end{tabular}

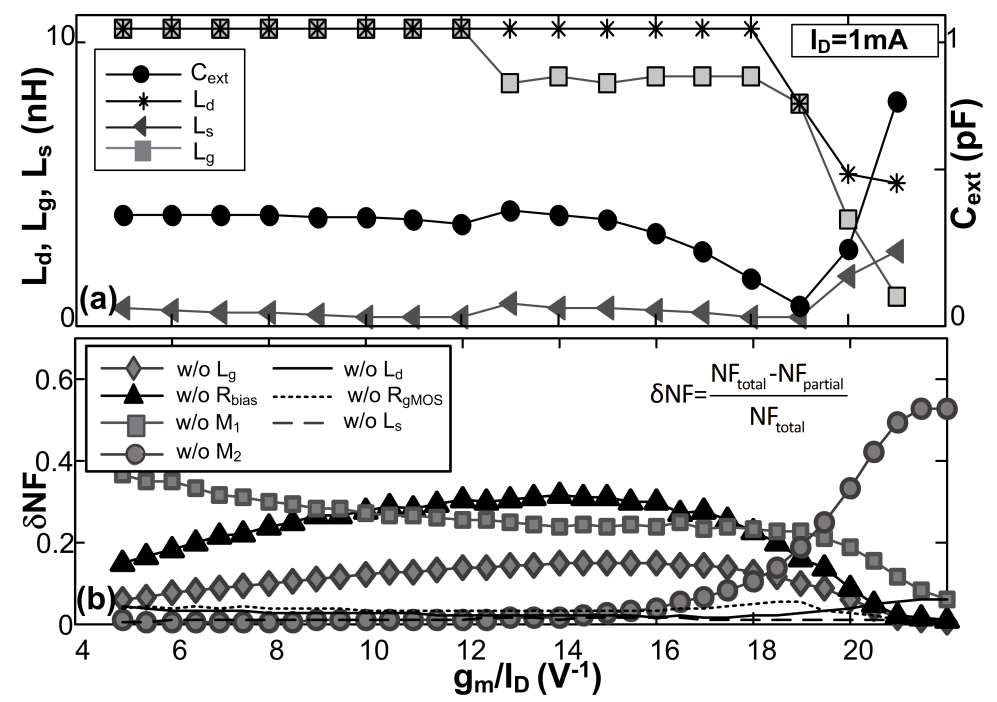

Fig. 6. (a) Inductors and $C_{e x t}$ values for $I_{D}=1 \mathrm{~mA}$. (b) Relative error in NF if the corresponding noise source is not included. 


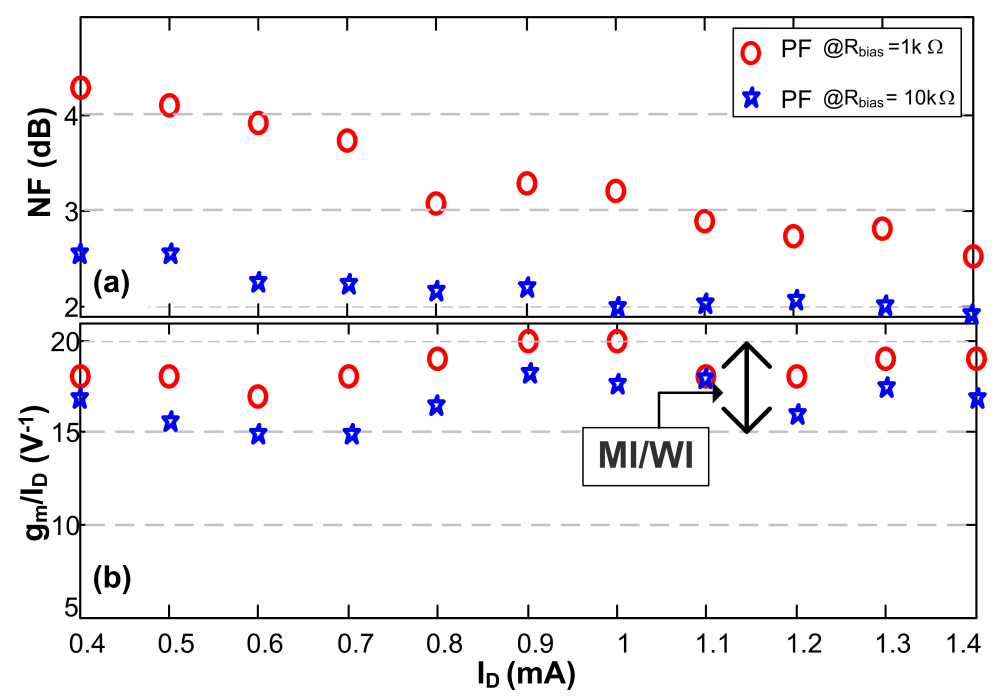

Fig. 7. Pareto optimal frontiers (PF) obtained for two different $R_{\text {bias }}$ values with the proposed EOP: (a) optimal NF and (b) optimal $g_{m} / I_{D}$.

Concerning LNA's components values, Fig. 6(a) shows the behavior of $L_{g}, L_{s}, L_{d}$ and $C_{e x t}$ when we move from SI to WI. Firstly, we observe that moving through MI-WI does not imply an increment in area. In this region, inductors $L_{g}$ and $L_{d}$-the most-demanding area components- are smaller than in SI, counteracting the MOST width increment. It occurs because, as shown in Fig 6.(b), the $N F$ contribution of cascode transistor $M_{2}$ increases considerably over $g_{m} / I_{D}=17 \mathrm{~V}^{-1}$, and as $M_{2}$ width is fixed, the unique possibility to reduce the noise is to reduce $R_{g}^{(p)}$, i.e. to decrease $L_{g}$. But as $Z_{i n}$ should be real, if $L_{g}$ decreases, $L_{s}$ and $C_{\text {ext }}$ must decrease.

The optimal designs with the minimum $N F$ (for high gain values) are observed in Fig. 7(a), concerning two very different $R_{\text {bias }}$ values. Despite the noticeable difference in values between the two $N F$ frontiers (almost $2 \mathrm{~dB}$ in SI), all optimal designs are in the MI-WI region, which covers $g_{m} / I_{D} \in[16,20] V^{-1}$, as visualized in Fig. 7(b). This fact proves that, even for such different $R_{b i a s}$ values, for the Reference Technology, moderate-weak inversion region is the optimum MOST biasing region for CS-LNAs for $2.4 \mathrm{GHz} .^{3}$

${ }^{3}$ For high $g_{m} / I_{D}$ ratios, above 21, the MOST is not in the quasistatic region and the MOST model here used is compromised. 


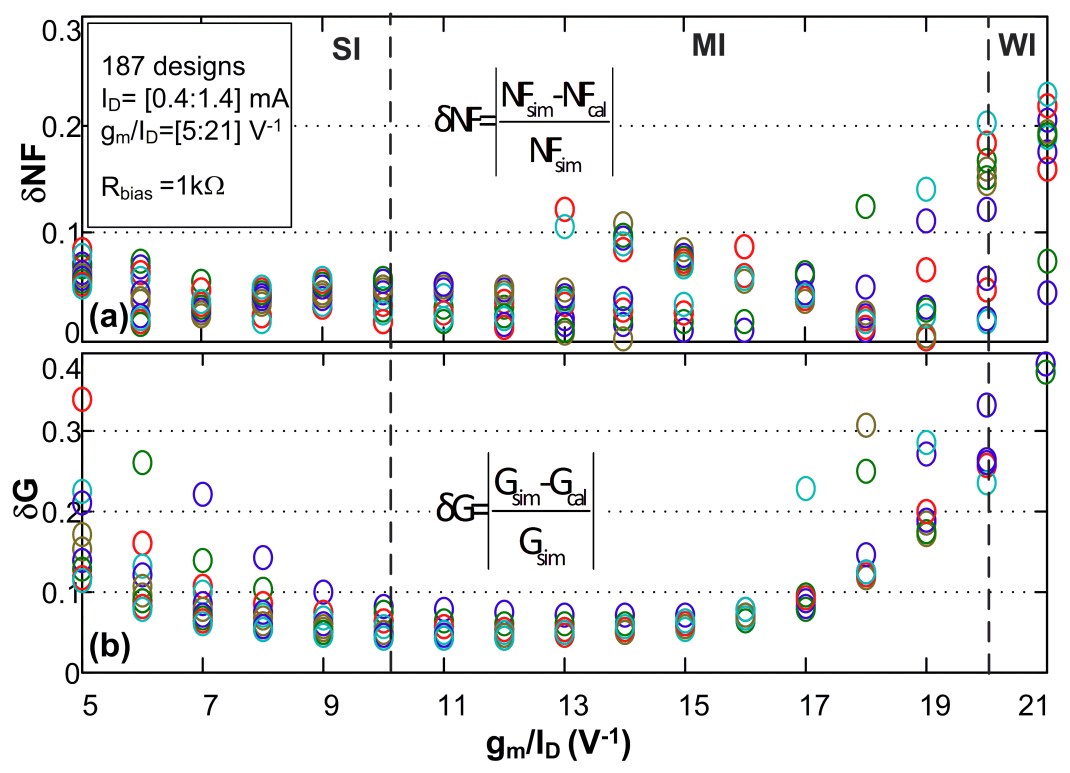

Fig. 8. (a) GdB and (b) NF relative errors in calculated versus simulated designs.

\section{A. Validation by simulation}

To validate the Pareto frontier found with the Iterative Procedure of Section III, for all-inversion regions and for the conditions of frequency, load and source previously defined, and $R_{b i a s}=1 \mathrm{k} \Omega$, we firstly vary $g_{m} / I_{D} \in[5,21] \mathrm{V}^{-1}$ and $I_{D} \in[0.4,1.4] \mathrm{mA}$, generating almost two hundred possible CS-LNA designs. Later, the designs are simulated exactly as obtained from computation, without any retouch. For each design, the computed characteristics of $G$ and $N F$ are compared with the results obtained from electrical simulations. The relative errors of $N F$ and $G$, plotted in Fig. 8, show that the used model describes very well the behavior up to MI-WI, and only for designs with very low power gains the relative error increase up to $30 \%$. Also, in MI-WI and WI regions relative errors are boosted due to the degradation of $S_{22}$. This degradation in MI-WI and WI occurs because the output matching is very sensitive to output LNA impedance values, which have an inherent error as they are computed theoretically. By doing a later adjustment in $C_{d 1}$ and $C_{d 2}$, these errors can be reduced. We consider that the errors observed are acceptable to validate the optimum zone presented in Fig. 7(b).

To provide a reduced set of characteristics of some of these designs, twelve CS-LNA are chosen, referred as $\{\mathrm{Si}, \mathrm{SMi}, \mathrm{Mi}, \mathrm{MWi}\}$, with $\mathrm{i}=\{1,2,3\}$ in Fig. 5 and Table I. Three current values are considered $(1.3 \mathrm{~mA}, 0.9 \mathrm{~mA}, 0.5 \mathrm{~mA})$ as well as four possible inversion regions $\left(g_{m} / I_{D}\left(V^{-1}\right)=6(\mathrm{SI}), 11\right.$ (SI-MI), 16 (MI) up to 19 (MI-WI)). Table I presents the LNA components sizes, noise figure and power gain 
obtained via the EOP with no constraints, for these twelve design points. Each LNA design is simulated via SpectreRF, acquiring $S_{11}, S_{22}, N F, G$ and $I I P 3$.

\section{B. Discussion}

Figure 7, supported with the validation of Section IV.A, shows that LNA optimal designs are in the MI/WI region. The use of all-inversion regions (SI, MI and WI) of the MOST is mandatory in this study because only in this way the MI-WI region is proven as the optimum design zone.

Following the procedure of Section IV, the optimum design zone can be for other processes and other LNA schemes [11], as the method only needs a small-signal modeling. This methodology also allows the easy visualization of the design compromises, providing beforehand a complete panorama and insight of the LNA behaviour when bias current and inversion level are modified.

The good results obtained in terms of noise figure or gain in all-inversion regions, particularly in moderate and weak inversion are because of having considered the following effects in the CS-LNA modeling: (1) simple semi-empirical MOST model covering all-inversion region; (2) noise parameters modeled in MOST all-inversion regions; (3) inclusion of the effects of all components to compute input/output impedance, in special all MOST capacitances; and (4) the inclusion of the cascode transistor effects.

\section{EXPERIMENTAL VALIDATION}

The experimental validation of the optimum design zone is done by presenting an untrimmed 2.4-GHz differential CS-LNA implemented in the Reference Technology to be used in a fully differential ZigBee receiver. The results that will be shown are of the first integration of this stand-alone prototype. For this implementation, the total power consumption and noise figure cannot surpass the $1.8 \mathrm{~mW}$ (with $V_{D D}=1.2 \mathrm{~V}$ ) and $5 \mathrm{~dB}$ respectively. The gain $G$ must be higher than $10 \mathrm{~dB}$ and the IIP3 higher than $-5 \mathrm{dBm}$. The ABCD network is considered to be the blocking capacitor and the probing pad RF model. Due to area constraints, Rbias is reduced up to $1 \mathrm{k} \Omega$. This allows to place $R_{\text {bias }}$ near $M_{1}$ and reduce considerably the layout parasitics in its MOST gate. Finally, to design a differential circuit based on the proposed method, we obtain the single-ended design following the EOP of Section IV; then we mirror the circuit to generate a differential structure. In our differential LNA we employ a differential symmetric source inductor with its center tap connected to ground; since the $L_{s}$ calculated by the procedure is single ended, we use the double of this value to find a differential inductor included in the technology set. This 
TABLE II

Comparison between Matlab and SpectreRF Simulation DATA FOR THE IMPLEMENTED CS-LNA AT $2.45 \mathrm{GHz}$.

\begin{tabular}{|l|cc|}
\hline Characteristics & Calculated & Post-layout \\
\hline$\left(g_{m} / I_{D}, I_{D}\right)\left(V^{-1}, \mathrm{~A}\right)$ & \multicolumn{2}{|c|}{$(17.4,570 \mu)$} \\
$L_{s}, L_{g}, L_{d}(\mathrm{nH})$ & $0.76,8.8,10.5$ & $0.75,11.1,10.5$ \\
$W_{M_{1}}(\mu m)$ & 92.5 & 92 \\
$C_{e x t}(\mathrm{fF})$ & 300 & 240 \\
$C_{d 1}, C_{d 2}(\mathrm{pF})$ & $0.38,1.35$ & $0.34,0.53$ \\
\hline $\mathrm{G}(\mathrm{dB})$ & 12 & 10.3 \\
$\mathrm{NF}(\mathrm{dB})$ & 3.9 & 4.3 \\
$\mathrm{IIP} 3, \mathrm{P} 1 \mathrm{~dB}, \mathrm{IIP} 2(\mathrm{dBm})$ & $-7^{\mathrm{a}}, \mathrm{n} / \mathrm{a}, \mathrm{n} / \mathrm{a}$ & $-3.5,-15,61$ \\
$\mathrm{~S}_{11}, \mathrm{~S}_{22}(\mathrm{~dB})$ & $-50,-21.5$ & $-27,-15$ \\
\hline
\end{tabular}

a Obtained from electrical simulation

procedure was not done for $L_{d}$ as the double of the computed values were not feasible in the Reference Technology.

After running the EOP, the implemented design is chosen among all the ones included in the Pareto frontier that have the minimum power consumption while achieving the above specifications. We consider all those designs whose $I I P 3$, estimated by electrical simulation of the schematic, are greater than a bound of $-7.5 \mathrm{dBm}$, near below the specified limit of $-5 \mathrm{dBm}$ to cover inaccuracies in the IIP3 computation. The optimal designs that fulfill this requirement are the ones with $I_{D}>0.5 \mathrm{~mA}$. Feature $I I P 3_{\min }$ is only imposed to the post-layout representation due to its high sensitivity to gain variations. In addition, as we need to consider those designs with power consumption restricted to $1.8 \mathrm{~mW}$ or less, a fine tuning using a smaller grid of $I_{D}$ in the range of $[0.5,0.8] \mathrm{mA}$ is performed. The condition imposed is to reduce as much as possible the power consumption while fulfilling the IIP3 requirement; the power consumption of the final single-ended design is $684 \mu \mathrm{W}$. Table II lists the chosen design characteristics as well as the post-layout simulations, which reach a good matching. Sizing adjustments of $L_{g}$ and $C_{e x t}$ in the post-layout simulation are due to the compensation of layout parasitics of the routing and the pads capacitances. The reduction in the final gain is because of the increment of parasitic resistances, which in turn, increase the final IIP3.

Final schematic and the microphotograph of the implemented LNA are shown in Fig. 9; it covers 


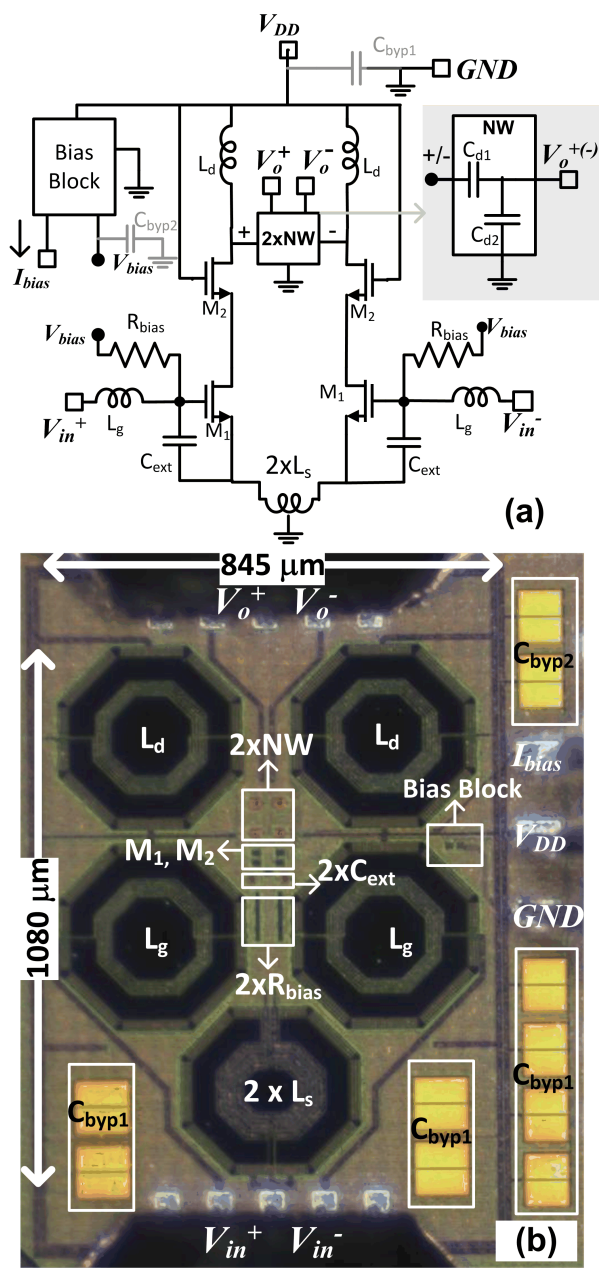

Fig. 9. Fabricated CS-LNA: (a) schematic and (b) microphotograph. Active area, passive components and output network are highlighted.

$0.91 \mathrm{~mm}^{2}(845 \mu \mathrm{m} \times 1080 \mu \mathrm{m})$ without considering pads area. LNA characterization was made on bare dies, using a Cascade RF microprobe station. S-parameter measurements were performed with the Agilent N5230 Two-ports Network Analyzer. Noise figure was measured with the Agilent N8974A Noise Figure Analyzer. As this equipment is single-ended, we were able to do only single-ended measurements on each one of two halves of the differential LNA, inferring, by symmetry, a similar behaviour for the differential scheme. Power gain and IIP3 were measured with the Agilent E4440A Spectrum Analyzer in differential mode with the aid of external baluns.

Post-layout simulated and setup-corrected S-parameters are shown in Fig. 10 in the $2.0 \mathrm{GHz}-2.8 \mathrm{GHz}$ band. Input and output networks resonant frequencies have a shift down of $180 \mathrm{MHz}$ and $110 \mathrm{MHz}$, 


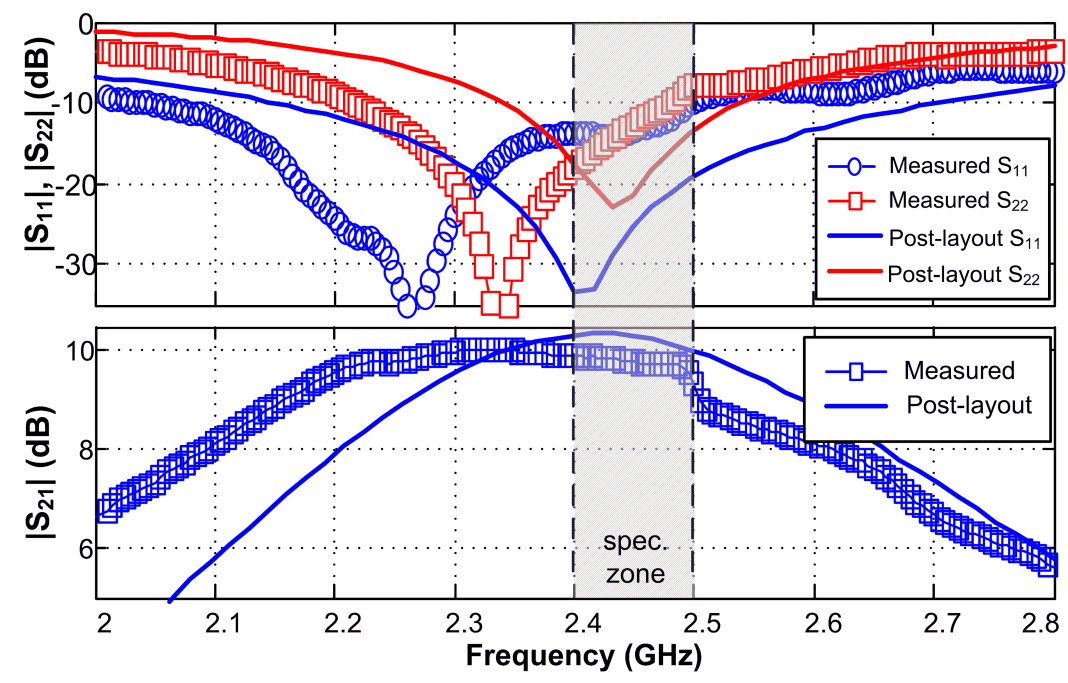

Fig. 10. Measured S-parameters of the fabricated CS-LNA: $\left|S_{11}\right|$ and $\left|S_{22}\right|$ (above), and $\left|S_{21}\right|$ (below). The ZigBee band is shadowed.

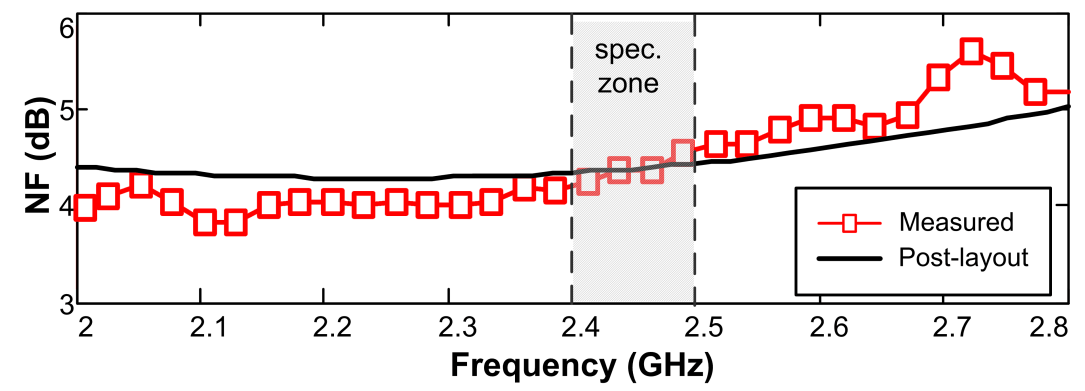

Fig. 11. Noise Figure measurements in the $2.0 \mathrm{GHz}-2.8 \mathrm{GHz}$ band, where the ZigBee band is shadowed.

respectively, visualized where traces $S_{11}$ and $S_{22}$ are minimal. These shifts, expected from performed corners simulations, reduce power gain and increase noise figure in the band of interest. LNA gain is equal to $9.7 \mathrm{~dB}$ at $2.45 \mathrm{GHz}$; the maximum gain is $10 \mathrm{~dB}$, only $0.3 \mathrm{~dB}$ lower than the typical post-layout simulation. LNA isolation is correct, as $\left|S_{12}\right|$ is below $-35 \mathrm{~dB}$ (not shown).

Figure 11 depicts the noise figure in the $2.0 \mathrm{GHz}-2.8 \mathrm{GHz}$ band, with a minimum of $3.9 \mathrm{~dB}$. At $2.45 \mathrm{GHz}$, it achieves $4.36 \mathrm{~dB}$, very near of the expected value.

The third and second order intermodulation points (IIP3, IIP2) as well as the 1-dB compression point $(P 1 d B)$ were measured. Fig.12 plots the measured amplitude of the fundamental and third order intermodulation spur when two tones separated by $1 \mathrm{MHz}$, with variable amplitude, were injected. Extrapolating, the IIP3 value is $-4 \mathrm{dBm}$. The inset of Fig.12 is a sample of the output spectrum of 


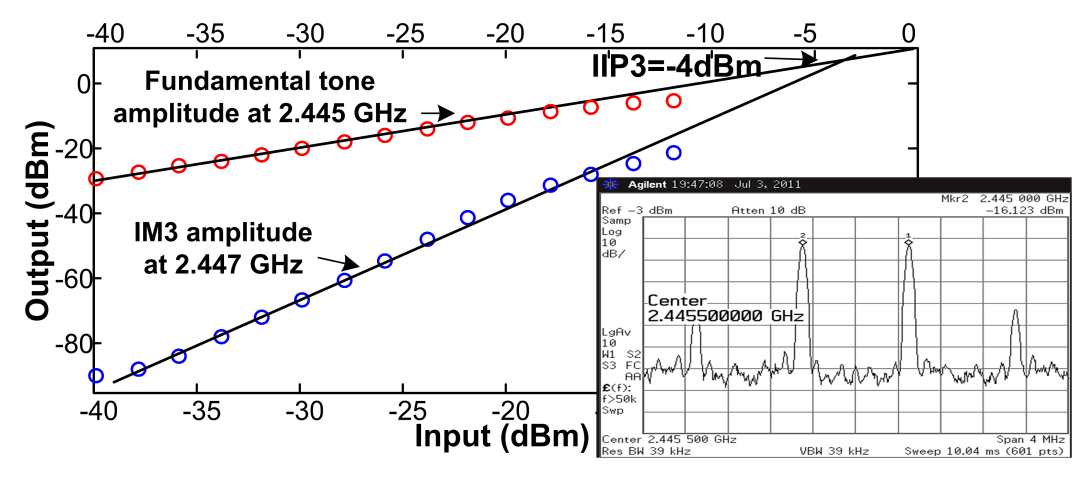

Fig. 12. Measured IIP3 for the fabricated circuit and output spectrum of the principal and IM3 tones (inset).

TABLE III

PERFORMANCE COMPARISON OF RECENTLY PUBLISHED CS-LNAS.

\begin{tabular}{|l|c|c|c|c|c|c|c|}
\hline Source & $\begin{array}{c}\text { FoM } \\
(\mathrm{dB})\end{array}$ & $\begin{array}{c}P_{D C} \\
(\mathrm{~mW})\end{array}$ & $\begin{array}{c}\mathrm{NF} \\
(\mathrm{dB})\end{array}$ & $\begin{array}{c}\text { IIP3 } \\
(\mathrm{dBm})\end{array}$ & $\begin{array}{c}\mathrm{G} \\
(\mathrm{dB})\end{array}$ & $\begin{array}{c}\text { Tech. } \\
(\mathrm{nm})\end{array}$ & $\begin{array}{c}f_{0} \\
(\mathrm{GHz})\end{array}$ \\
\hline T.W. $^{\mathrm{a}}$ & 8.9 & 0.68 & 4.36 & -4 & 9.7 & 90 & 2.45 \\
\hline$[27]$ & 6.7 & 1.08 & 3.1 & -21.4 & 25 & 130 & 2.3 \\
{$[7]$} & 3.9 & 0.4 & 4.7 & -11 & 9.1 & 130 & 3 \\
{$[28]$} & 3.8 & 3.8 & 2.2 & -16 & 20.8 & 130 & 2 \\
{$[8]$} & 2.8 & 0.95 & 5.2 & -19 & 21.4 & 180 & 2.4 \\
{$[29]$} & -0.15 & $0.49^{\mathrm{a}}$ & 5.2 & -19 & 15.5 & 180 & 2.45 \\
{$[30]$} & -0.95 & $1.08^{\mathrm{b}}$ & 9 & -10 & 14 & 180 & 2.4 \\
\hline
\end{tabular}

a Single-ended structure considered to normalize the comparison.

${ }^{\mathrm{b}}$ Inferred from authors.

the two-tone input signals. Finally the input second order intermodulation point (IIP2) was measured by injecting two tones at $2.445 \mathrm{GHz} \pm 1.25 \mathrm{MHz}(2.44625 \mathrm{GHz}$ and $2.44375 \mathrm{GHz})$, so the second order intermodulation tone falls in $2.5 \mathrm{MHz}$ within the receiver baseband. Extrapolating, the IIP2 value is 61 $\mathrm{dBm}$. Finally, The P1dB measure was about $-15 \mathrm{dBm}$ (not shown).

Table III presents the performance comparison of our designed moderate inversion CS-LNA with that of some prior low-power works, utilizing the usual figure of merit that includes the effect of the IIP3, $F o M=10 \log \left(\left(G_{l i n} \cdot I I P 3 \cdot f\right) /\left(\left(10^{N F / 10}-1\right) \cdot P_{D C}\right)\right)[26]$; where $G_{l i n}$ is the power gain in W/W, IIP3 is in $\mathrm{mW}, f$ is the working frequency in $\mathrm{GHz}, N F$ is the noise figure in $\mathrm{dB}$, and $P_{D C}$ is the total power in $\mathrm{mW}$. As observed, our CS-LNA is excellently positioned. 


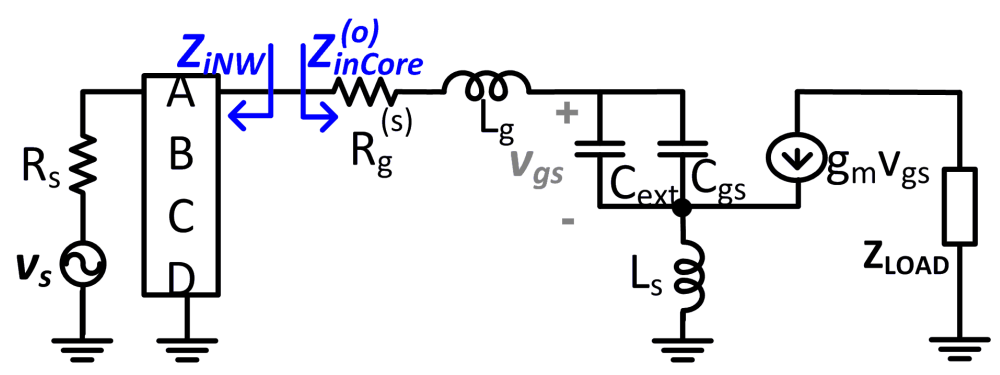

Fig. 13. Simplified scheme of the LNA to compute the initial values of $Z_{i n}$.

\section{CONCLUSiOnS}

In this paper it is shown that the optimum design zone for $90-\mathrm{nm}$ CS-LNAs at $2.4 \mathrm{GHz}$ is the moderateweak inversion region. It is achieved by applying a noise figure-power consumption optimization technique for RF CS-LNA for nanometer technologies based on the $g_{m} / I_{D}$ technique, which can be extended to other LNAs architectures with only a correct small-signal model of the complete circuitry. Both MOS transistor and inductor data are extracted with SpectreRF simulations to accurately and quickly model them, including their actual behavior in the design flow. Effects of choke resistor $R_{\text {bias }}$ have been included and outlined when small and high values are used. As a proof of concept, an application example is implemented. Following the proposed method, the mathematical computation of noise figure and power gain as well as electrical simulations and measurements accord with each other with very good error levels. Little re-design is needed after the computed results.

\section{APPENDIX}

\section{A. Evaluation of approximate initial values of $C_{\text {ext }}$ and $L_{s}$}

When we need to determine the network in Fig. 3 by means of numerical resolution of the equation system: $Z_{i n}\left(C_{e x t}, L_{s} ; f_{0}\right)=R_{s}$, with unknowns $C_{e x t}$ and $L_{s}$, it is necessary to give a set of initial values close enough to actual solution so that convergence is better and faster. We obtain this initial solution from a very simplified network depicted in Fig. 13. Amplifier MOST $M_{1}$ has been reduced to its simplest transconductor model with gate-source capacitance. Cascode transistor $M_{2}$ and output stage have been combined in $Z_{L O A D}$ impedance. Choke resistor $R_{\text {bias }}$ has not been considered. To simplify the evaluation and formulas, we will move the matching condition to the second port of the ABCD network and we will use the serial parasitic resistance $R_{g}^{(s)}$ of gate inductor $L_{g}$. This way, we have the following equation 
system:

$$
Z_{\text {inCore }}^{(o)}\left(C_{\text {ext }}, L_{s} ; f_{0}\right)=Z_{i N W}^{*}\left(f_{0}\right)
$$

where the asterisk indicates the complex conjugate operator.

For this simple network, the $Z_{\text {inCore }}$ impedance is:

$$
\begin{aligned}
Z_{\text {inCore }}^{(o)}\left(f_{0}\right) & =\left.\frac{1+s\left(R_{g}^{(s)} C_{t}+g_{m} L_{s}\right)+s^{2} C_{t} L_{t}}{s C_{t}}\right|_{s=j \omega_{0}} \\
& =\left(R_{g}^{(s)}+\frac{g_{m}}{L_{s} C_{t}}\right)+j\left(\frac{\omega_{0}^{2} C_{t} L_{t}-1}{\omega_{0} C_{t}}\right)
\end{aligned}
$$

where $C_{t}=C_{g s}+C_{e x t}, L_{t}=L_{g}+L_{s}$. The output impedance of ABCD network is: $Z_{i N W}\left(f_{0}\right)=$ $\left(B+D R_{s}\right) /\left(A+C R_{s}\right)$; if we write it this way: $Z_{i N W}\left(f_{0}\right)=R_{i i}+j \omega_{0} L_{i i}$, with $L_{i i}$ positive, negative or null value, but always $R_{i i}>0$, the equation system (4) is:

$$
\begin{aligned}
R_{i i} & =R_{g}^{(s)}+\frac{g_{m}}{L_{s} C_{t}} \\
\omega_{0} L_{i i} & =\left(1-\omega_{0}^{2} C_{t} L_{t}\right) /\left(\omega_{0} C_{t}\right) .
\end{aligned}
$$

Solving this one,

$$
\begin{aligned}
C_{t} & =\frac{g_{m}}{2 R_{i g}}\left(\sqrt{L_{i g}^{2}+\frac{4 R_{i g}}{g_{m} \omega_{0}^{2}}}-L_{i g}\right) \\
L_{s} & =\frac{R_{i g} C_{t}}{g_{m}} \\
C_{\text {ext }} & =C_{t}-C_{g s}
\end{aligned}
$$

where $R_{i g}=R_{i i}-R_{g}^{(s)}$ and $L_{i g}=L_{i i}+L_{g}$.

\section{B. Noise figure evaluation}

Noise figure of CS-LNA in Fig. 3 is calculated as in [31]:

$$
N F_{C S-L N A}=10 \log \left(F_{C S-L N A}\right)=10 \log \left(\frac{N_{o}-N_{o, L o a d}}{N_{o, v s}}\right)
$$

where $N_{o}-N_{o, \text { Load }}$ is the total noise due to all noise sources at the output voltage $v_{o}$ on the load $R_{L}$ excluding the noise of this one, and $N_{o, v s}$ is the noise at output voltage $v_{o}$ due to only the input source, that is $R_{s}$. 
Since we have considered only five main noise sources: choke resistor, $R_{b i a s}$, gate and drain inductors, $L_{g}, L_{d}$, and transistors $M_{1}$ and $M_{2}$, the following expression for noise factor is:

$$
\begin{aligned}
& F_{C S-L N A}=1+
\end{aligned}
$$

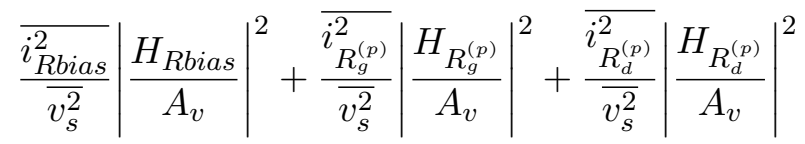

$$
\begin{aligned}
& +\overline{\frac{i_{R_{g M O S}}^{2}}{\overline{v_{s}^{2}}}}\left|\frac{H 1_{R_{g M O S}}}{A_{v}}\right|^{2}+\left.\overline{\overline{i_{n d}^{2}}} \frac{H 1_{i d}}{\overline{v_{s}^{2}}}\right|^{2}+\overline{\frac{i_{n g}^{2}}{\overline{v_{s}^{2}}}}\left|\frac{H 1_{i g}}{A_{v}}\right|^{2} \\
& +\frac{\overline{i_{n g} i_{n d}^{*}}}{\overline{v_{s}^{2}}} \frac{H 1_{i g} H 1_{i d}^{*}}{\left|A_{v}\right|^{2}}+\frac{\overline{i_{n g}^{*} i_{n d}}}{\overline{v_{s}^{2}}} \frac{H 1_{i g}^{*} H 1_{i d}}{\left|A_{v}\right|^{2}}+\frac{\overline{i_{n d}^{2}}}{\overline{v_{s}^{2}}}\left|\frac{H 2_{i d}}{A_{v}}\right|^{2}
\end{aligned}
$$

where the noise psd due to $R_{s}$ is $\overline{v_{s}^{2}}=4 k_{B} T R_{s}$, and for the other resistances is $\overline{i_{R x}^{2}}=4 k_{B} T / R_{x}$, with $R_{x}=\left\{R_{\text {bias }}, R_{d}^{(p)}, R_{g}^{(p)}, R_{g M O S}\right\}$. Models for drain current white-noise psd, $\overline{i_{n d}^{2}}$, and gate induced current noise psd, $\overline{i_{n g}^{2}}$, of amplifier MOS transistor are in Section II. The cross correlation terms are assumed as in

[12], $\overline{i_{n g} i_{n d}^{*}}=-\overline{i_{n g}^{*} i_{n d}}=j|c| \sqrt{\overline{i_{n g}^{2} i_{n d}^{2}}}$, where $|c| \cong 0.4$. For amplifier MOST, $M_{1}$, we have considered all its noise sources, but for cascode MOST, $M_{2}$, only the drain white noise contribution has been applied, disregarding induced gate noise and the correlated terms. Each one of these sources provides a contribution at the output through the transfer function from the noise source to the output voltage; for $R_{s}$ transfer function is the total voltage gain, $A_{v}=v_{o} / v_{s}$ and for the other noisy current sources are $H_{x}=v_{o} / i_{x}$. To distinguish transfer functions corresponding to transistors' sources, a numerical index has been added, $H_{1 x}$ and $H_{2 x}$ for $M_{1}$ and $M_{2}$ respectively. Obviously, all these transfer functions are available when the whole the linear circuit has been solved.

\section{Proposed optimization flow}

This section details the Exhaustive Optimization Process (EOP), which is the global procedure we implement to obtain the Pareto-optimal design frontier, as discussed in the rest of the paper. The procedure makes use of all resources considered in previous sections: technological database of active and passive RF devices (Section II) and CS-LNA small-signal model described in Section III and Appendices A and B. We implement the optimization process as an exhaustive search method in the full design domain of $I_{D}$ and $g_{m} / I_{D}$. The EOP is sketched in the flow diagram of Fig. 14 and conceptually represented in Fig. 15.(a); it is performed on a fixed grid of the design domain: $\Psi_{g m / I D} \times \Psi_{I D}=\left\{\left(g_{m} / I_{D}\right)_{k}\right\} \times$ $\left\{I_{D, i}\right\} \subset\left[\left(g_{m} / I_{D}\right)_{\min },\left(g_{m} / I_{D}\right)_{\max }\right] \times\left[I_{D, \min }, I_{D, \max }\right]$.

Fixing some procedure constraints (MOST length, interface and bias resistances and minimum capacitor value, i.e.: $L_{\min }, R_{L}, R_{s}, R_{\text {bias }}$ and $C_{\text {min }}$ ) and specification limits (maximum noise figure $N F_{\max }$, 


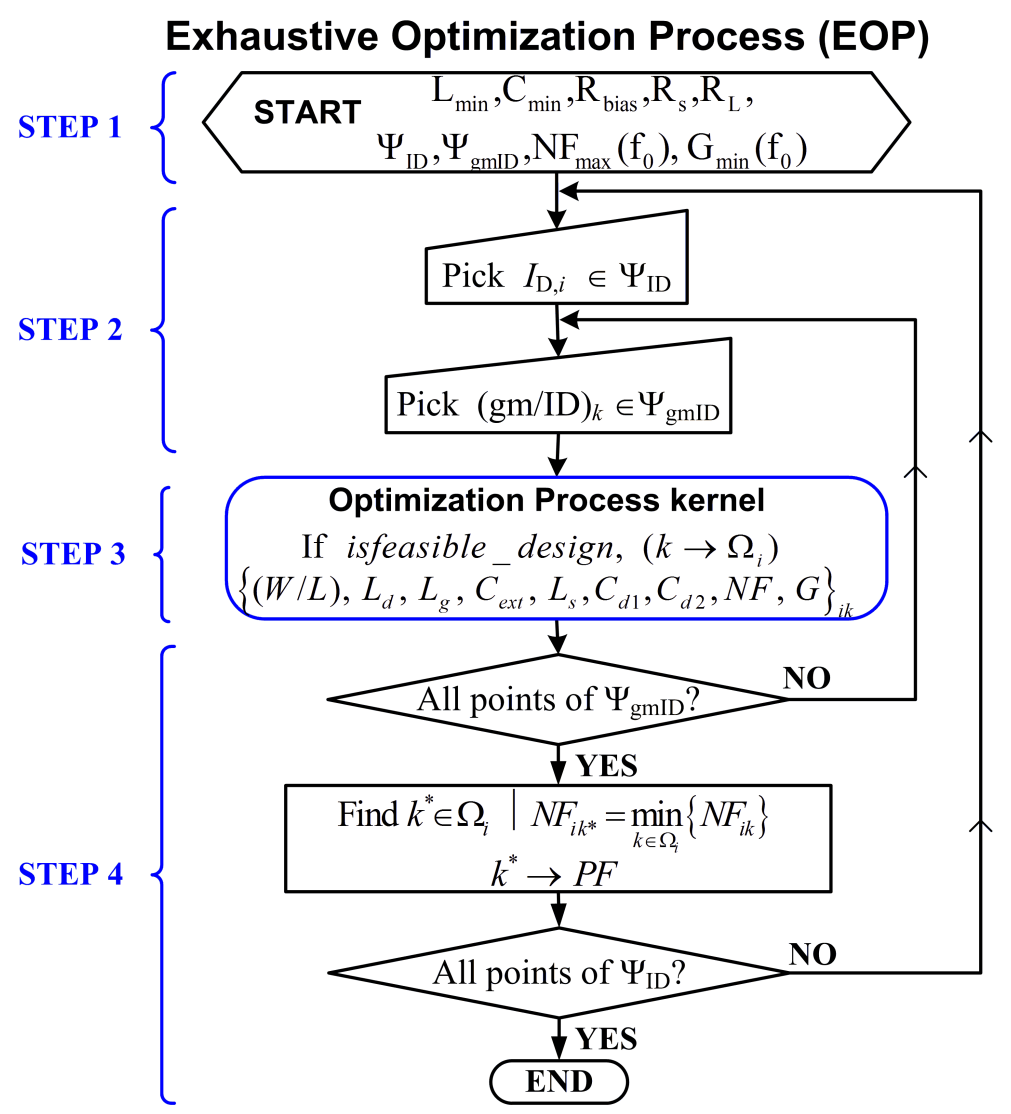

Fig. 14. Flow diagram of the proposed CS-LNA optimization process.

minimum power gain $G_{m i n}$ at $f_{0}$ ), for each drain current $I_{D, i}$ of $\Psi_{I D}$, the process kernel finds all designs in $\Psi_{g m / I D}$ compatible with the limits of noise figure $\left(\leq N F_{\max }\right)$, power consumption $\left(\leq I_{D, \max }\right)$ and power gain $\left(\geq G_{\min }\right)$, which are gathered in $\Omega_{i}$. Restriction on power consumption is implicitly verified because of the construction of search grid. In the set $\Omega_{i}$ of compatible designs with $I_{D, i}$, we select the one $\left(k^{*}\right)$ with the minimum noise figure $\left(N F_{i k^{*}}\right)$ for the Pareto trade-off curve, saved in the Pateto-frontier collection (PF). Constraints on CS-LNA linearity $\left(I I P 3_{\text {min }}\right)$ are considered at the end of the optimization process by electrically simulating the reached design ${ }^{4}$.

The key points of process kernel are: for each possible gate inductor $L_{g}$ included in the LUT $\Lambda_{L}$ we find its corresponding LNA and its characteristics. Then, among all $L_{g}$, we find the one that minimizes

${ }^{4}$ Precise IIP3 analytical expressions for all inversion region of a CS-LNA as the one depicted in Fig. 3 is a non-solved task nowadays [32]. 

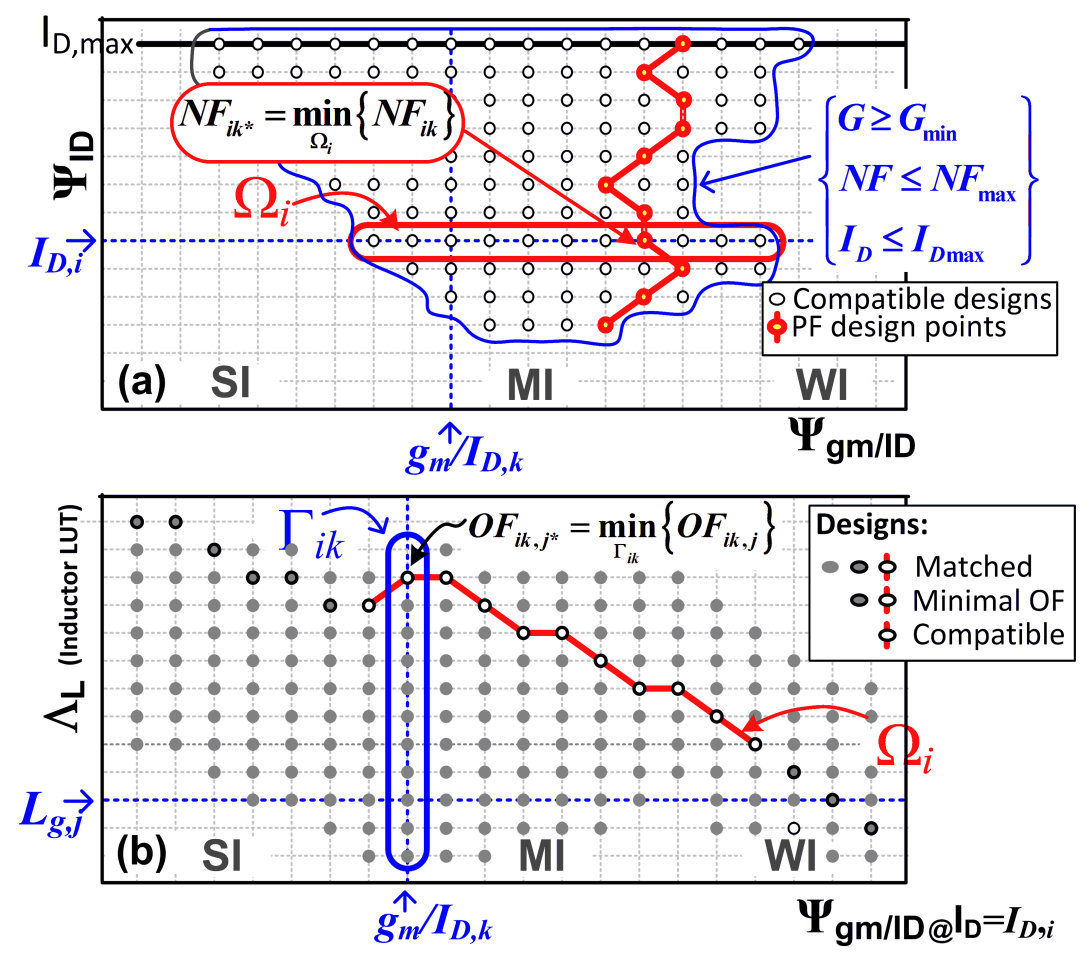

Fig. 15. Conceptual representation of outputs and sets involved in (a) EOP and (b) EOP kernel.

the objective function $O F=N F-G$, while verifying all constraints ${ }^{5}$. If two or more designs provide the same minimum $O F$, area restriction is applied to discriminate a unique solution. If no design verifies the established constraints, the kernel considers as unfeasible the given inversion level $\left(g_{m} / I_{D}\right)_{k}$ for the entered drain current $I_{D, i}$.

Now consider in detail the EOP kernel (see Fig. 15.(b)). Its input is the pair $\left(\left(g_{m} / I_{D}\right)_{k}, I_{D, i}\right)$ of the design domain $\left[\left(g_{m} / I_{D}\right)_{\min },\left(g_{m} / I_{D}\right)_{\max }\right] \times\left[I_{D, \min }, I_{D, \max }\right]$. Other magnitudes and LUTs needed are globally defined in the full EOP. The process starts considering that each inductor, $L_{i n d, j}$ of $\Lambda_{L}$ is the gate inductor $L_{g, j}$, and then $C_{e x t, j}$ and $L_{s, j}$ are computed following the Iterative Procedure of Section III. If a matched design is achieved, noise figure $N F_{j}$ and power gain $G_{j}$ are computed with (8)-(9) and (3), and the design of index $j$ is saved in the $\Gamma_{i k}$ collection.

When all inductors have been tested, we select the design $j^{*}$ in $\Gamma_{i k}$ with minimal OF and minimal area. Then if noise figure and gain of this design satisfies the constraints $\left(N F_{j^{*}} \leq N F_{\max }\right.$ and $\left.G_{j}^{*} \geq G_{\min }\right)$, the kernel indicates to the EOP with a logical flag, isfeasible_design=1, that in this inversion level there

\footnotetext{
${ }^{5}$ The objective function is very similar to the FoM of [26] with a fixed $I_{D}$, excluding the $I I P 3$ characteristic.
} 
is a compatible design, and the kernel outputs this design too.

\section{REFERENCES}

[1] D. Comer and D. Comer, "Operation of analog MOS circuits in the weak or moderate inversion region," IEEE Transactions on Education, vol. 47, no. 4, pp. 430-435, 2004.

[2] A.-S. Porret and et al., "An ultralow -power UHF transceiver integrated in a standard digital CMOS process: Arquitecture and receiver," IEEE Journal of Solid-State Circuits, vol. 36, no. 3, pp. 452-464, Mar. 2001.

[3] J. Ramos and et al, “90nm RF CMOS technology for low-power 900MHz applications," Proceeding of the 34th European Solid-State Device Research conference ESSDERC 2004, pp. 329-332, Sep. 2004.

[4] L. Barboni, R. Fiorelli, and F. Silveira, "A tool for design exploration and power optimization of CMOS RF circuit blocks," IEEE International Symposium on Circuits and Systems ISCAS'06, May 2006.

[5] A. Shameli and P. Heydari, "A novel power optimization technique for ultra-low power RFIDs," in International Symposium on Low Power Electronics and Design, G. Tegernsee, Ed., 2006.

[6] H. Lee and S. Mohammadi, "A subthreshold low phase noise CMOS LC VCO for ultra low power applications," IEEE Microwave and Wireless Component Letters, vol. 17, no. 11, pp. 796-799, Nov. 2007.

[7] — , "A 3GHz subthreshold CMOS low noise amplifier," in Proc. IEEE Radio Frequency Integrated Circuits (RFIC) Symp, 2006.

[8] A. V. Do, C. C. Boon, M. A. Do, K. S. Yeo, and A. Cabuk, "A subthreshold low-noise amplifier optimized for ultralow-power applications in the ISM band," IEEE Transactions on Microwave Theory and Techniques, vol. 56, no. 2, pp. 286-292, 2008.

[9] H.-S. Jhon and et al, "0.7 V supply highly linear subthreshold low-noise amplifier design for 2.4GHz wireless sensor network applications," Microwave And Optical Technology Letters, vol. 51, no. 5, pp. 1316-1320, May 2009.

[10] R. Fiorelli, E. Peralías, and F. Silveira, "LC-VCO design optimization methodology based on the $g_{m} / I_{D}$ ratio for nanometer CMOS technologies," IEEE Transactions on Microwave Theory and Techniques, vol. 59, no. 7, pp. 1822-1831, Jul. 2011.

[11] R. Fiorelli and F. Silveira, "Common gate LNA design space exploration in all inversion regions," in Argentine School of Micro-Nanoelectronics, Technology and Applications, EAMTA., 2008, pp. 119-122.

[12] D. Shaeffer and T. H. Lee, "A 1.5-V, 1.5-GHz CMOS low noise amplifier," IEEE Journal of Solid-State Circuits, vol. 32, no. 5, pp. 745-759, May 1997.

[13] T.-K. Nguyen, C.-H. Kim, G.-J. Ihm, M.-S. Yang, , and S.-G. Lee, "CMOS low-noise amplifier design optimization techniques," IEEE Transactions on Microwave Theory and Techniques, vol. 52, no. 5, pp. 1433-1442, May 2004.

[14] L. Belostotski and J. W. Haslett, "Noise figure optimization of inductively degenerated CMOS LNAs with integrated gate inductors," IEEE Transactions on Circuits and Systems, vol. 53, no. 7, pp. 1409-1422, Jul 2006.

[15] P. Andreani and H. Sjöland, "Noise optimization of an inductively degenerated CMOS low noise amplifier," IEEE Transactions on Circuits and Systems, vol. 48, no. 9, pp. 835-841, Sept 2001.

[16] J. Janssens and M. Steyaert, CMOS Celular Receiver Front-ends. Kluwer Academic Publishers, 2002.

[17] G. Tulunay and S. Balkir, "A synthesis tool for CMOS RF low-noise amplifiers," IEEE Transactions on Computer-Aided Design of Integrated Circuits and Systems, vol. 27, no. 5, pp. 977-982, 2008.

[18] A. Nieuwoudt, T. Ragheb, H. Nejati, and Y. Massoud, "Numerical design optimization methodology for wideband and multi-band inductively degenerated cascode CMOS Low Noise Amplifiers," IEEE Transactions on Circuits and Systems I, vol. 56, no. 6, pp. 1088-1101, 2009. 
[19] N. Barabino, R. Fiorelli, and F. Silveira, "Efficiency based fesign for fully-integrated Class C RF Power Amplifiers in nanometric CMOS," in Proceedings of IEEE International Symposium in Circuits and Systems, ISCAS 2010, May 2010.

[20] Z. Deng and A. M. Niknejad, "On the noise optimization of CMOS common-source low-noise amplifiers," IEEE Transactions on Circuits and Systems-Part I: Fundamental Theory and Applications, vol. 58, no. 4, pp. 654-667, 2011.

[21] F. Silveira, D. Flandre, and P. G. A. Jespers, "A $g_{m} / I_{D}$ based methodology for the design of CMOS analog circuits and its applications to the synthesis of a silicon-on-insulator micropower OTA," IEEE Journal of Solid-State Circuits, vol. 31, no. 9, pp. 1314-1319, Sep. 1996.

[22] P. G. Jespers, The gm/ID Methodology, a sizing tool for low-voltage analog CMOS Circuits. Springer, 2010.

[23] A.Cunha, M. C. Schneider, and C. Galup-Montoro, “An MOS transistor model for analog circuit design,” IEEE Journal of Solid-State Circuits, vol. 33, no. 10, pp. 1510-1519, Oct. 1998.

[24] Y. Tsividis, Operation and Modelling of the MOS Transistor, 2nd ed. Oxford University Press, 2000.

[25] R. Fiorelli, F. Silveira, A. Rueda, and E. Peralías, "Semi-empirical model of MOST and passive devices focused on narrowband RF blocks," in XXVII Conference on Design of Circuits and Integrated Systems (DCIS), Nov. 2012.

[26] R. Brederlow, W. Weber, J. Sauerer, S. Donnay, P. Wambacq, and M. Vertregt, "A mixed-signal design roadmap,” IEEE Design Test of Computers, vol. 18, no. 6, pp. $34-46$, nov/dec 2001.

[27] F. Tzeng, A. Jahanian, and P. Heydari, “A multiband inductor-reuse CMOS low-noise amplifier," IEEE Transactions on Circuits and Systems II, vol. 55, no. 3, pp. 209-213, 2008.

[28] J. Borremans, P. Wambacq, C. Soens, Y. Rolain, and M. Kuijk, "Low-area active-feedback low-noise amplifier design in scaled digital CMOS," IEEE Journal of Solid-State Circuits, vol. 43, no. 11, pp. 2422 -2433, Nov. 2008.

[29] T. Tran, C. Boon, M. Do, and K. Yeo, "Ultra-low power series input resonance differential common gate LNA," Electronics Letters, vol. 47, no. 12, pp. 703 -704, Sep. 2011.

[30] A. V. Do, C. C. Boon, M. A. Do, K. S. Yeo, and A. Cabuk, "An energy-aware CMOS receiver front end for low-power 2.4-GHz applications," IEEE Transactions on Circuits and Systems I, vol. 57, no. 10, pp. 2675-2684, 2010.

[31] B. Razavi, RF Microelectronics. Prentice Hall Inc., 1998.

[32] B. Toole, C. Plett, and M. Cloutier, "RF circuit implications of moderate inversion enhanced linear region in MOSFETs," IEEE Transactions on Circuits and Systems I, vol. 51, no. 2, pp. 319 - 328, Feb. 2004. 